\title{
Lebens- und Sterbewünsche bei unheilbarer Krankheit
}

Roland Kunz

Korrespondenz:

Dr. med. Roland Kunz Spital Affoltern CH-8910 Affoltern am Albis

roland.kunz[at]spitalaffoltern.ch
In der Diskussion um das Thema der organisierten Suizidbeihilfe wird immer wieder die politische Stärkung der Palliative Care als Lösung empfohlen oder gefordert. Dadurch entsteht gewollt oder ungewollt eine Polarität zwischen Palliative Care und Suizidbeihilfe. Diese können und dürfen sich aber nicht gegenseitig ersetzen und sollen deshalb nebeneinander betrachtet und nicht einander gegenübergestellt werden

Beide Konzepte versuchen, leidenden Menschen Hilfe anzubieten, jedoch mit völlig unterschiedlichem Ansatz. Die Suizidbeihilfe ist auf das Beenden des Lebens, auf Sterbehilfe, fokussiert. Die Palliativmedizin oder besser die Palliative Care als interdisziplinäres und integratives Behandlungskonzept stellt das Leben mit einer Krankheit und die Lebensqualität des unheilbaren Patienten ins Zentrum. Sie fokussiert auf Lebenshilfe, akzeptiert aber die Grenzen des Lebens und der Medizin und damit das Sterben des Patienten. Sie orientiert sich nicht am Kampf gegen die Krankheit, sondern an den individuellen Bedürfnissen des Patienten. Was sind aber diese Bedürfnisse? Sie lassen sich mit den vier «S» umschreiben: Symptomlinderung, Selbstbestimmung, Sicherheit und Support.

\section{Symptomlinderung}

Viele Menschen fürchten ihr natürliches Ende im Rahmen einer fortschreitenden Krankheit, weil sie Angst vor unbehandelbaren Schmerzen, vor einem
Die Schweizerische Akademie der Medizinischen Wissenschaften (SAMW) hat 2004 medizin-ethische Richtlinien zur «Betreuung von Patientinnen und Patienten am Lebensende» veröffentlicht. Anfragen an die Zentrale Ethikkommission (ZEK) der SAMW haben gezeigt, dass in Einzelfällen eine nicht vertretbare Praxis der ärztlichen Suizidhilfe besteht, und zwar teilweise mit, teilweise aber auch ohne Beteiligung einer Sterbehilfeorganisation. Die ZEK hat dies zum Anlass genommen, in einer Stellungnahme auf die in den Richtlinien aufgeführten Kriterien hinzuweisen und die Voraussetzungen für deren Einhaltung zu präzisieren. Die Stellungnahme wurde in der SÄZ 11/2012 vom 14. März 2012 veröffentlicht.

In dieser und einigen folgenden Ausgaben der SÄZ erscheinen kurze «Standpunkte», welche die Thematik der ärztlichen Suizidhilfe aus einer individuell-fachlichen Perspektive beleuchten.

Ärzte wirksam den Lebenswillen unserer Patienten unterstützen. Erstaunlich oft überwiegt noch unsere Angst, mit Opiaten das Leben zu verkürzen. Und mit dem folglich zögerlichen Einsatz wirksamer Medikamente fördern wir den Sterbewunsch der Patienten.

Lebenshilfe umfasst aber mehr als nur die Symptomlinderung, sie fordert eine Fokussierung auf das

\section{Gelingt eine rasche Linderung der Symptome, erleben wir häufig einen neu aufkeimenden Lebenswillen.}

terminalen Ersticken oder anderen unerträglichen Symptomen haben. Beim Eintritt in eine Palliativstation hören wir oft als Ziel der Patienten, möglichst bald sterben zu können. Bisher nur ungenügend gelinderte Symptome führen zur Angst, dass alles nur noch schlimmer werden kann, und diese letzte Lebensphase soll deshalb so kurz wie möglich dauern. Gelingt aber eine rasche Linderung der Symptome, erleben wir häufig wieder einen neu aufkeimenden Lebenswillen mit neuen Zielsetzungen. Mit einer Verbesserung der Behandlung von Schmerzen und anderen Symptomen können wir
Leiden der ganzen Person. Das heisst, dass wir den betroffenen Menschen als Einheit von Körper, Seele und Geist mit seinem mitbetroffenen Umfeld wahrnehmen und unterstützen.

\section{Selbstbestimmung}

Um ihre Autonomie leben zu können, brauchen schwerkranke Menschen umfassende Informationen über Prognose, mögliche Komplikationen, Behandlungsoptionen und -grenzen sowie Unterstützungsmöglichkeiten. Nur wer die Möglichkeiten und Grenzen der Versorgungsorte kennt, kann 


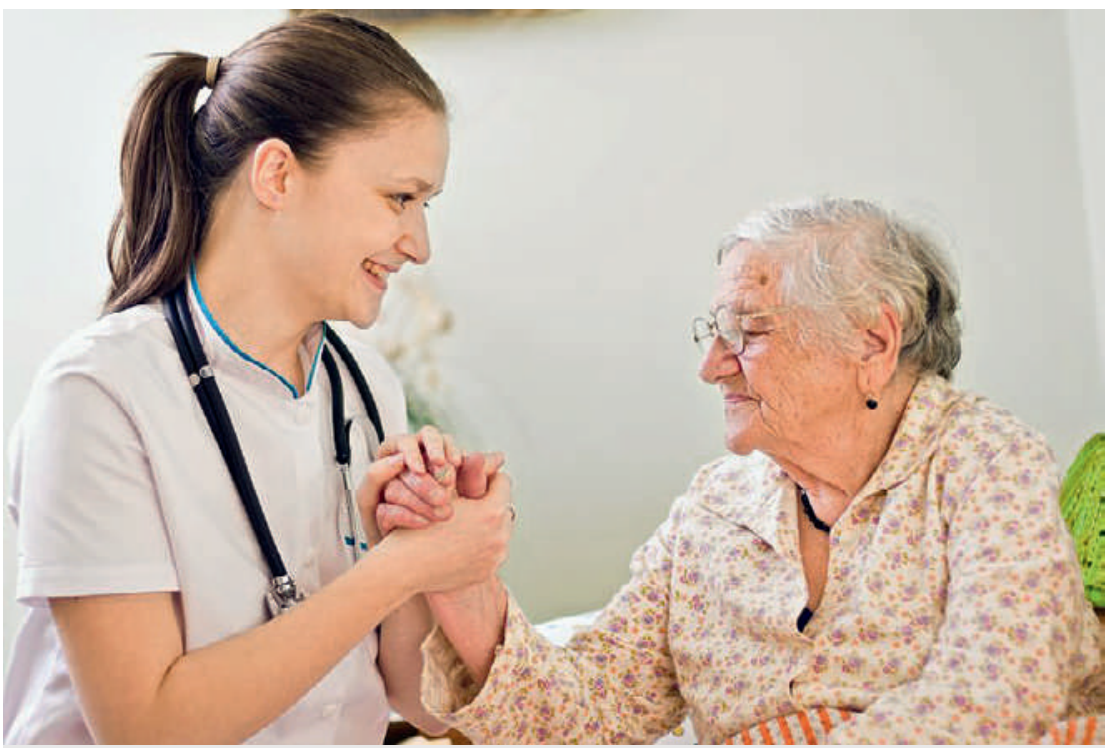

Das Vertrauen auf ärztliche Linderung von Schmerzen nimmt Angst und kann den Sterbewunsch in den Hintergrund schieben. hung zum behandelnden Arzt - sowohl Hausarzt wie Spitalarzt - ist entscheidend für das Vertrauen. Wenn der Patient mit seiner unheilbaren Krankheit Vertrauen hat, dass die behandelnden Ärzte seinen Willen respektieren, dass auch bei allfälliger Kommunikationsunfähigkeit sein dokumentierter Wille weiterhin vollumfänglich beachtet wird, wächst die Bereitschaft, das Kommende geschehen zu lassen und zu akzeptieren. Die Sicherheit, dass bei einer Zunahme von Schmerzen rasch wirksame Linderung möglich ist oder bei Akutsituationen wie Atemnot oder Blutungen eine Sedation möglich ist, kann den Sterbewunsch in den Hintergrund schieben. Damit steigt auch die Offenheit, die verbleibenden Tage bewusst zu leben und nicht nur das Ende herbeizuwünschen. Dies erfordert aber unser Antizipieren von möglichen Problemsituationen durch Instruktion der Pflegenden, der Angehörigen und entsprechende Bereitstellung der notwendigen Medikamente. Die Handynummer des Hausarztes und der Spitex auf dem Nachttisch kann viel zum Sicherheitsgefühl beitragen.

\section{Sicherheit hemmt Angst, auch Angst vor dem Sterben.} wie weit lebensverlängernde Massnahmen weitergeführt werden sollen und wo er seine Grenzen setzt. Das Festhalten des persönlichen Willens in einer aktuellen Patientenverfügung hilft ihm, Ängste zu vermindern.

Bei vielen Krankheiten kann der Verlauf nur ungenau vorausgesehen werden, und Entscheidungen müssen immer wieder neu getroffen werden. Es gibt aber durchaus Diagnosen, bei denen die Betroffenen sehr konkret absehen können, was auf sie zukommt. So setzen sich beispielsweise Patienten mit einer ALS im Krankheitsverlauf wiederholt mit entscheidenden Fragen auseinander: Möchte ich eine PEG, möchte ich bis zur Ateminsuffizienz warten und dann beatmet werden, will ich die immer grösser werdende Abhängigkeit ertragen? Die Palliativmedizin kann bei der fortgeschrittenen ALS zwar begleiten, die Symptome lindern, bei einer terminalen Ateminsuffizienz auch eine Sedation anbieten. Aber die Möglichkeiten der «Lebenshilfe» bleiben sehr beschränkt. So erstaunt es nicht, dass bei keiner anderen Diagnose so häufig der Wunsch nach einer selbstbestimmten Beendigung des Lebens aufkommt. Unsere Aufgabe wird es dann, den Patienten und seine Angehörigen in der Entscheidung zu begleiten und zu beraten. Wie weit wir uns schliesslich aktiv an einer Umsetzung beteiligen, ist ein ganz persönlicher Gewissensentscheid.

\section{Sicherheit}

Sicherheit hemmt Angst, auch Angst vor dem Sterben. Sicherheit beruht auf Vertrauen. Die Bezie-

\section{Support}

Unterstützung bei unheilbarer Krankheit umfasst einerseits professionelle Angebote wie Sozialberatung zur Lösung finanzieller Nöte und rechtlicher Probleme, psychologische Begleitung von Patient und Umfeld, seelsorgerliche Begleitung in existentiellen Fragen, andererseits Akzeptanz in Krankheit und Abhängigkeit durch die Gesellschaft. Wertschätzung zu erleben trotz Ausscheiden aus dem Arbeitsprozess, nicht als kostensteigernder Faktor im Gesundheitswesen wahrgenommen werden, sondern als wertvolles Glied unserer Gesellschaft, sind Beiträge im Sinne der Lebenshilfe zur Verminderung des Sterbewunsches.

\section{Fazit}

Flächendeckende Angebote der Palliative Care, Integration der entsprechenden Kenntnisse in die Aus- und Weiterbildung und gesellschaftliche Akzeptanz von Krankheit und Abhängigkeit statt Überbetonung von Leistung und Unabhängigkeit sind zentrale Voraussetzungen, um den Lebenswunsch und -willen trotz unheilbarer Krankheit zu stützen. Erst wenn wir mit gutem Gewissen sagen können, dass diese Ziele erreicht sind, können wir auch mit gutem Gewissen Suizidbeihilfe für Patienten mit persistierendem Sterbewunsch unterstützen. 